binding protein-1 as a key transcription factor for nutritional induction of lipogenic enzyme genes. J. Biol. Chem. 274:35832-35839.

11. Bergot, M.O., Diaz-Guerra, M.J., Puzenat, N., Raymondjean, M., and Kahn, A. 1992. Cis-regulation of the L-type pyruvate kinase gene promoter by glucose, insulin and cyclic AMP. Nucleic Acids Res. 20:1871-1877.

12. Shih, H.M., Liu, Z., and Towle, H.C. 1995. Two CACGTG motifs with proper spacing dictate the carbohydrate regulation of hepatic gene transcription. J. Biol. Chem. 270:21991-21997.

13. Burgess, S.C., et al. 2008. Carbohydrate-response element-binding protein deletion alters substrate utilization producing an energy-deficient liver. J. Biol. Chem. 283:1670-1678.

14. Nishimura, M., Fedorov, S., and Uyeda, K. 1994. Glucose-stimulated synthesis of fructose 2,6bisphosphate in rat liver. Dephosphorylation of fructose 6-phosphate, 2-kinase:fructose 2,6bisphosphatase and activation by a sugar phosphate. J. Biol. Chem. 269:26100-26106.

15. Kabashima, T., Kawaguchi, T., Wadzinski, B.E., and Uyeda, K. 2003. Xylulose 5-phosphate mediates glucose-induced lipogenesis by xylulose 5 -phosphate-activated protein phosphatase in rat liver. Proc. Natl. Acad. Sci. U. S. A. 100:5107-5112.

16. Zelcer, N., and Tontonoz, P. 2006. Liver X recep- tors as integrators of metabolic and inflammatory signaling. J. Clin. Invest. 116:607-614.

17. Repa, J.J., and Mangelsdorf, D.J. 2002. The liver X receptor gene team: potential new players in atherosclerosis. Nat. Med. 8:1243-1248.

18. Cha, J.Y., and Repa, J.J. 2007. The liver X receptor (LXR) and hepatic lipogenesis. The carbohydrateresponse element-binding protein is a target gene of LXR. J. Biol. Chem. 282:743-751.

19. Tobin, K.A., et al. 2002. Liver X receptors as insulin-mediating factors in fatty acid and cholesterol biosynthesis. J. Biol. Chem. 277:10691-10697.

20. Lazar, M.A., and Willson, T.M. 2007. Sweet dreams for LXR. Cell Metab. 5:159-161.

\title{
Growth hormone resurrects adult human thymus during HIV-1 infection
}

Kiki Tesselaar and Frank Miedema

Department of Immunology, University Medical Center Utrecht, Utrecht, The Netherlands.

In conditions of severe $T$ cell depletion, such as HIV-1 infection, limited T cell production by the thymus can thwart the immune response, putting individuals at increased risk of infection with opportunistic pathogens. In this issue of the JCI, Napolitano et al. report, in a prospective, randomized study, that treatment of HIV-1-infected adults with growth hormone may reverse thymic atrophy, as reflected by increased de novo thymic $T$ cell production accompanied by increased peripheral $T$ cell production (see the related article beginning on page 1085). While the long-term immunological and clinical benefits of growth hormone treatment remain unclear, the data suggest a way in which to enhance thymopoiesis and peripheral $T$ cell production in immunodeficient individuals.

$\mathrm{T}$ cell depletion due to (a) chemo- or radiotherapy for the treatment of cancer; (b) myeloablative chemotherapy prior to bone marrow transplantation; (c) immunosuppressive treatment to avoid transplant rejection; or (d) HIV infection can be quite prolonged. These patients may be susceptible to life-threatening viral infections and opportunistic and virus-induced cancers for longer periods of time. Moreover, given the increasing numbers of aged individuals in the human population, age-related loss of immune function is a significant and growing clinical problem. Loss of thymus function and decreasing de novo naive $T$ cell production during aging may be the main cause for failing immunity in the elderly $(1,2)$.

Nonstandard abbreviations used: GH, growth hormone; HAART, highly active antiretroviral therapy; rGH, recombinantGH; TRA@, TCR $\alpha$ locus; TRD@, TCR $\delta$ locus; TREC, TCR rearrangement excision circle.

Conflict of interest: The authors have declared that no conflict of interest exists.

Citation for this article: J. Clin. Invest. 118:844-847 (2008). doi:10.1172/JCI35112.
Peripheral, homeostatic $\mathrm{T}$ cell proliferation may compensate for decreasing and insufficient thymic production of naive $\mathrm{T}$ cells but does not broaden the TCR repertoire. Since for proper $\mathrm{T}$ cell reconstitution, influx of recent thymic emigrants into the $T$ cell pool is required to maintain the naive and memory $T$ cell pool and the TCR repertoire, homeostatic proliferation may result in accelerated aging of the $\mathrm{T}$ cell pool, as reflected by limited TCR diversity, loss of $\mathrm{T}$ cell function, and cellular senescence (3).

Loss of thymic export as a predominant source of $\mathrm{T}$ cell neogenesis with age has been formally proven in the mouse (1). In humans, the association between thymic atrophy and the decline of naive $\mathrm{T}$ cell numbers and TCR rearrangement excision circle (TREC) frequency with age is well documented (1). TRECs are stable DNA circles that are excised from $\mathrm{T}$ cell germline DNA to allow for TCR formation during early $\mathrm{T}$ cell development. Briefly, early in $\mathrm{T}$ cell neogenesis within the thymus, during TCR generation and rearrangement, the TCR $\delta$ locus (TRD@) is excised from within the TCR $\alpha$ locus (TRA@). This excision generates a circular episomal DNA fragment known as a TREC (Figure 1). Thymic emigrants have high concentrations of TRECs, which upon cell division are not replicated but are gradually diluted with every round of division. Increase in TREC density and number has become an accepted indicator of thymic function. Thymic output has been found to be directly related to thymic cellularity (4), but in the mouse there is no evidence for rebound of thymic output as a homeostatic response to severe depletion of the peripheral $\mathrm{T}$ cell pool. In humans, evidence for thymic rebound has been claimed based on increased thymic mass or exceedingly high TREC frequencies observed during immune reconstitution. However, this issue remains controversial (5).

\section{Causes of thymic atrophy}

Gradual, age-associated thymic atrophy is believed to be caused by declining levels of several hormones, including human growth hormone $(\mathrm{GH})$ and its mediator IGF-1, as well as the cytokine IL-7 (1). In the mouse, a relationship between IL-7 and thymic T cell output, as well as between IL-7 and peripheral T cell division, are well established (6). Studies in humans and nonhuman primates, however, have mainly shown effects of IL-7 on peripheral $\mathrm{T}$ cell division (7-9).

$\mathrm{GH}$ is produced by a large variety of cell types, including thymocytes, thymic 


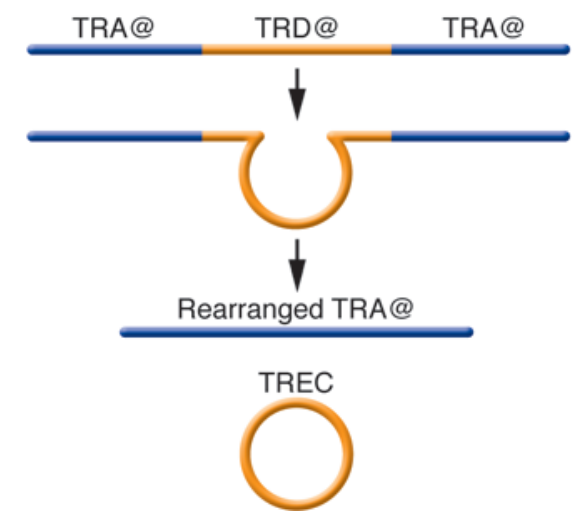

epithelial cells, and mature lymphocytes. Administration of $\mathrm{GH}$ and $\mathrm{GH}$-inducing compounds or proteins, including ghrelin, to enhance or restore thymic histological architecture, cellularity, and function has been studied mainly in the mouse (10). Interestingly, these studies show that under normal steady-state conditions, GH and IGF-1 are not required for and do not have a positive effect on $\mathrm{T}$ cell development or thymic output but do have a beneficial effect under conditions of stress such as viral infection, inflammation, $\mathrm{T}$ cell depletion, and thymic atrophy (7). In mice, GH administration increased thymic cellularity but was also found to be antiapoptotic and to promote $\mathrm{T}$ cell proliferation in recipients of allogeneic HSC transplants (11).

Due to the existence of clinical conditions characterized by slow $\mathrm{T}$ cell production rates, therapeutic approaches that restore thymic function through administration of GH, IGF-1, or IL-7, or combinations of these three or other agents, are highly desirable. As of this writing, only short-term clinical studies in the mouse have been performed (1), and little data are available on the effects of GH and IGF-1 on the immune system in humans, for which each has mainly been used for the treatment of children with short stature (12). Lack of clinical trials may be related to the very short half-life of recombinant $\mathrm{GH}(\mathrm{rGH})$. Daily injections are required and long-term treatment may be necessary in order to have a significant impact on the $\mathrm{T}$ cell pool. Lack of clinical trials may also be related to the well-documented adverse effects of GH treatment, including arthralgia and glucose intolerance.

In this issue of JCI, Napolitano et al. report, in an elegant and well-carriedout prospective, randomized, open-label study, a clinical trial with 22 HIV-1-infect-

\section{Figure 1}

Generation of TRECs. During thymopoiesis, the genes encoding the variable and constant regions of the TCR- $\alpha$ chain are being rearranged. This is accompanied by the deletion of the interspersed TRD@ and the formation of an episomal DNA excision circle (TREC) which can be detected and quantified by PCR.

ed adult patients receiving highly active antiretroviral therapy (HAART) who also received daily subcutaneous injections of rGH for six months (13). Previously, these authors examined the effects of $\mathrm{rGH}$ on the thymus of HIV-1-infected adults who were receiving $\mathrm{GH}$ as part of a lipodystrophy study $(14,15)$. In this earlier study, Napolitano et al. observed increased thymic mass and moderately increased numbers of naive $\mathrm{CD}^{+} \mathrm{T}$ cells, which led them to conclude that rGH administration had brought about a reversal of thymic atrophy and that de novo $T$ cell production could be induced (14). In the current JCI study (13), Napolitano et al. confirm and extend these previous studies, via the use of more refined $\mathrm{T}$ cell phenotyping and TREC analyses, in order to determine the source of the increase in naive $T$ cell numbers in these HIV-1-infected individuals receiving HAART and rGH (Figure 2).

\section{Mechanisms of GH-induced T cell renewal}

The major new finding in the current study (13) is that naive T cell gain in these individuals was not solely due to de novo thymic $\mathrm{T}$ cell production, but to a large extent derived from peripheral naive $\mathrm{CD}^{+}$ $T$ cell expansion. This is based on the finding that despite clear increases in thymic mass, in truly naive $\mathrm{CD} 4^{+} \mathrm{T}$ cell numbers, and in TREC frequencies in unfractionated PBMCs, a decrease in TREC frequencies in naive $\mathrm{CD}^{+} \mathrm{T}$ cells was observed. If all newly produced naive $\mathrm{CD} 4^{+} \mathrm{T}$ cells were recent thymic emigrants, with the characteristic relatively high TREC content, one would have expected clear increases in naive $\mathrm{CD} 4^{+} \mathrm{T}$ cell TREC frequencies, especially when baseline naive $\mathrm{T}$ cell counts were low (5) (Figure 2B). On the other hand, if the naive $\mathrm{CD}^{+} \mathrm{T}$ cell gains were completely due to periph- eral $\mathrm{T}$ cell expansion, this would have diluted the TREC content in naive $\mathrm{CD}^{+}$ $\mathrm{T}$ cells and PBMCs (Figure 2C). Thus, the observed effect of rGH on naive $\mathrm{CD}^{+} \mathrm{T}$ cell numbers and TREC content strongly suggests that both pathways of $\mathrm{T}$ cell production - de novo $\mathrm{T}$ cell production in the thymus and peripheral $\mathrm{T}$ cell division - contributed to the enhanced naive $\mathrm{T}$ cell generation in these individuals (Figure 2D) (16). These observed effects of rGH administration on peripheral $\mathrm{T}$ cell production are in line with the data obtained following GH administration in different murine models $(1,7)$.

In their current study, Napolitano et al. (13) emphasize the observed GH-associated increases in thymic mass, but since this increase was measured by CT scan, further evidence will need to be provided to confirm that this is the result of actively increased thymopoiesis. The concurrent increases in numbers of naive $T$ cells in the periphery are not necessarily due to an increased number of thymic emigrants, since it has been shown that increases in thymic mass and cellularity may derive from infiltrates of mature peripheral $\mathrm{T}$ cells and adipose tissue that fill the perivascular space (17).

Although the current analyses of the effects of rGH treatment are very extensive, future studies should include analysis of absolute increases in TREC numbers in order to corroborate the contribution of the thymus, as well as an analysis of the ratio of absolute naive $\mathrm{CD}^{+}$and $\mathrm{CD}^{+} \mathrm{T}$ cell gains, which if indeed thymus-derived should reflect the classical $\mathrm{CD}^{+} / \mathrm{CD}^{+}$ $\mathrm{T}$ cell ratio of $3: 1$ of recent thymic emigrants. Given that lack of TCR diversity is considered a reason for failing immunity in HIV-infected patients (18) and that only de novo $\mathrm{T}$ cell production from the thymus can increase TCR diversity, it will be essential to analyze the effect of GH on TCR repertoire in future studies. The contribution of peripheral $\mathrm{T}$ cell proliferation and survival might be further supported by measurement of Ki67, a cell cycle-related nuclear protein expressed by proliferating cells in all phases of the active cell cycle, and apoptosis measurements.

Napolitano et al. (13) clearly show that increased GH levels can improve thymic function, but these effects were only temporary. If GH treatment also promotes T cell survival, cessation of treatment might decrease $T$ cell lifespan and cause a drop in T cell numbers. Although it is not easy to deduce from the 

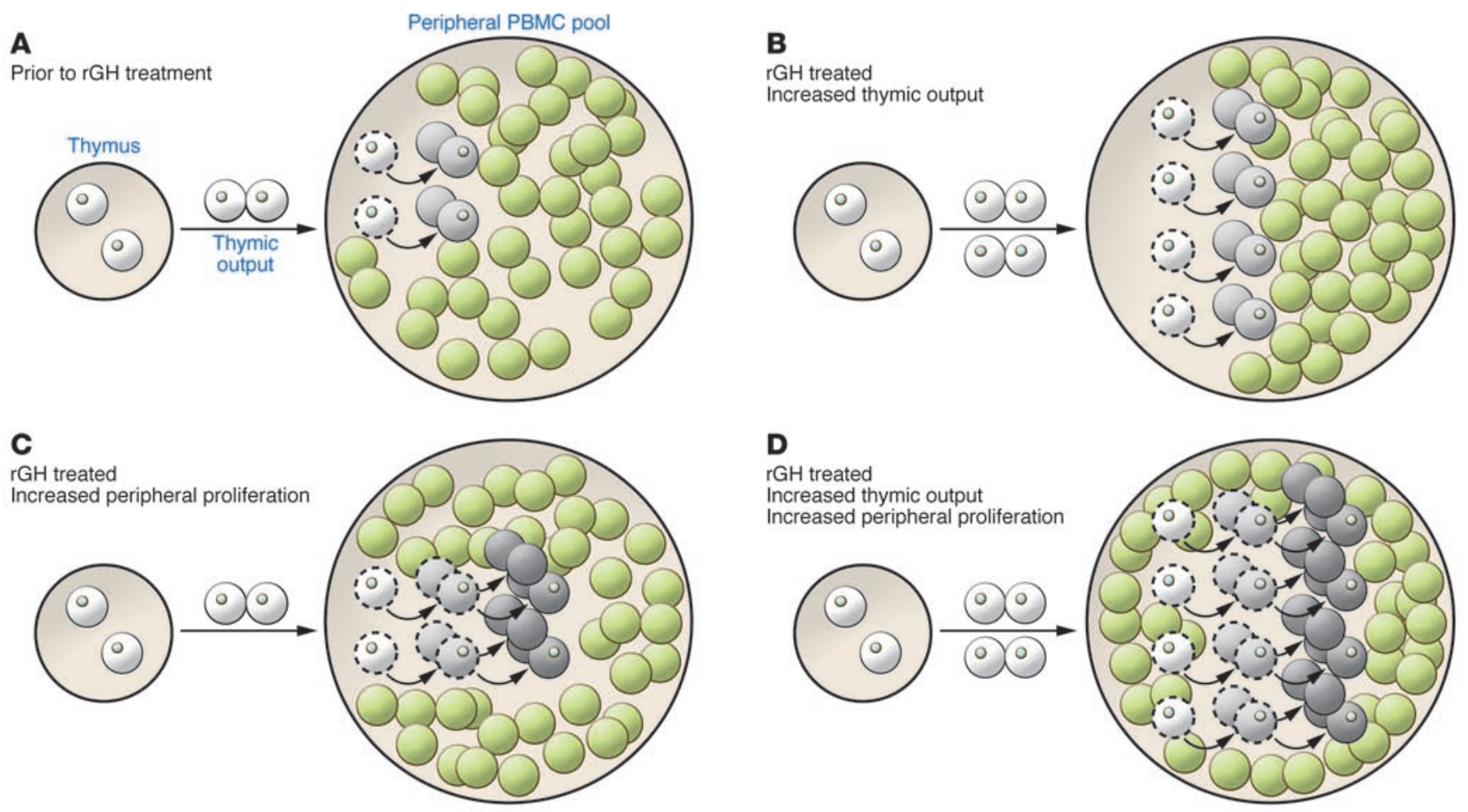

- TREC

Memory T cells, B cells, NK cells

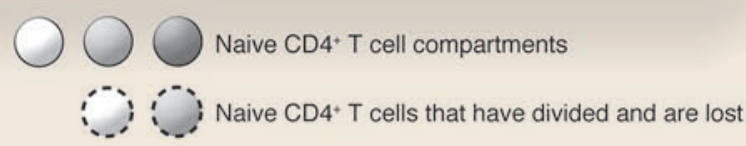

Figure 2

Elucidation of effects of rGH treatment on T cell and TREC dynamics in HIV-1-infected individuals. Effects of GH on thymic output and T cell proliferation have been described. Combined interpretation of T cell and TREC dynamics can be used to determine whether these effects contribute to increased naive CD4 ${ }^{+} \mathrm{T}$ cell numbers after $\mathrm{rGH}$ treatment in HIV-1-infected individuals. The figure exemplifies $\mathrm{T}$ cell dynamics (percentage of naive CD4+ ${ }^{+}$cells within the peripheral PBMC pool and number of naive CD4+ ${ }^{+}$cells) and TREC dynamics (TREC numbers and percentage of TREC-containing cells within the naive $\mathrm{CD} 4^{+} \mathrm{T}$ cell and PBMC population) prior to $\mathrm{GH}$ treatment $(\mathbf{A})$ and different possible scenarios following GH treatment (B-D). Prior to GH treatment (A), the thymic production of naive CD4+ $\mathrm{T}$ cells shown is 2 , and these cells divide 1 time in the periphery. This results in 4 naive CD4+ $T$ cells (gray), which in total contain 2 TRECs. In scenario $\mathbf{B}$, rGH is shown to only affect thymic output (4 naive $\mathrm{CD} 4{ }^{+} \mathrm{T}$ cells). In scenario $\mathbf{C}$, $\mathrm{rGH}$ is shown to only affect peripheral CD4+ $\mathrm{T}$ cell production (these cells are shown to divide twice in the periphery). In their current study in this issue of the $\mathrm{JCl}$, Napolitano et al. (13) show that in fact rGH administration to HAART-treated HIV-1infected adults concurrently affects both thymic output and peripheral naive CD4+ $T$ cell proliferation, as shown in scenario $D$. The resultant effects include increased thymic production of naive CD4+ $T$ cells and TRECs, an increase in the percentage of naive CD4 ${ }^{+} T$ cells and TRECs within the peripheral PBMC pool, but decreased TREC frequency within naive CD4+ $T$ cells.

reported data that the increase in peripheral $\mathrm{T}$ cell gain was sustained after cessation of $\mathrm{GH}$ treatment, the combined data suggest that this may indeed have been the case.

\section{Eligibility for GH treatment}

GH treatment involves daily injections and does carry a risk for serious side effects, and this therapeutic approach therefore calls for careful consideration. A better understanding of the underlying mechanisms of $\mathrm{T}$ cell gain will help identify patient groups that might be eligible and benefit most from this approach. Absolute TREC gain is regarded as the measure for thymic output, and it would thus be of interest to identify baseline predictors for absolute TREC gain. Although Napolitano et al. (13) report a larger gain in thymic density in patients with higher baseline $\mathrm{CD}^{+} \mathrm{T}$ cell counts or higher baseline IGF-1 levels, and a larger gain in thymic volume in patients with higher baseline GH levels, the meaning of these observations is unclear, since these data were not related to absolute TREC increases. Once they are identified, baseline predictors for absolute TREC gain, in combination with baseline $\mathrm{CD}^{+} \mathrm{T}$ cell numbers and time on HAART, may well turn out to be useful criteria for selection among HIV-infected patients of those individuals that may benefit most from GH therapy. Although it may be risky to extrapolate data obtained from HAARTtreated HIV-1-infected individuals to other patient groups, the suggested contribution of peripheral $\mathrm{T}$ cell proliferation to naive $\mathrm{T}$ cell generation provides a treatment opportunity for elderly patients, in whom thymic recovery might be reduced.

In conclusion, the current study of the effects of GH treatment in humans, which seems long overdue, provides encouraging evidence of increased $\mathrm{T}$ cell renewal (13). However, before wider clinical application can be considered, the mechanism 
and long-term immunological and clinical benefits of this approach need to be thoroughly investigated.

\section{Acknowledgments}

We thank Rogier van Gent, Mette Hazenberg, and Jose Borghans for their input.

Address correspondence to: Frank Miedema, University Medical Center Utrecht, Department of Immunology, Lundlaan 6, PO Box 85090, Utrecht UNK 3508 AB, The Netherlands. Phone: 31-88-755-7674; Fax: 31-30-250-4305; E-mail: f.miedema@ umcutrecht.nl.

1. Taub, D.D., and Longo, D.L. 2005. Insights into thymic aging and regeneration. Immunol. Rev. 205:72-93.

2. Clark, D.R., De Boer, R.J., Wolthers, K.C., and Miedema, F. 1999. T cell dynamics in HIV-1 infection. Adv. Immunol. 73:301-327.
3. Hakim, F.T., et al. 2005. Age-dependent incidence, time course, and consequences of thymic renewal in adults. J. Clin. Invest. 115:930-939.

4. Berzins, S.P., Boyd, R.L., and Miller, J.F.A.P. 1998. The role of the thymus and recent thymic migrants in the maintenance of the adult peripheral lymphocyte pool. J. Exp. Med. 187:1839-1848.

5. Hazenberg, M.D., Borghans, J.A., De Boer, R.J., and Miedema, F. 2003. Thymic output: a bad TREC record. Nat. Immunol. 4:97-99.

6. Fry, T.J., and Mackall, C.L. 2005. The many faces of IL-7: from lymphopoiesis to peripheral $\mathrm{T}$ cell maintenance. J. Immunol. 174:6571-6576.

7. van den Brink, M.R., Alpdogan, O., and Boyd, R.L. 2004. Strategies to enhance T-cell reconstitution in immunocompromised patients. Nat. Rev. Immunol. 4:856-867.

8. Storek, J., et al. 2003. Interleukin-7 improves CD4 T-cell reconstitution after autologous CD34 cell transplantation in monkeys. Blood. 101:4209-4218.

9. Sportes, C., and Gress, R.E. 2007. Interleukin-7 immunotherapy. Adv. Exp. Med. Biol. 601:321-333.

10. Dixit, V.D., et al. 2007. Ghrelin promotes thymopoiesis during aging. J. Clin. Invest. 117:2778-2790.

11. Murphy, W.J., and Longo, D.L. 2000. Growth hormone as an immunomodulating therapeutic agent.
Immunol. Today. 21:211-213.

12. Wit, J.M., Kooijman, R., Rijkers, G.T., and Zegers, B.J. 1993. Immunological findings in growth hormone-treated patients. Horm. Res. 39:107-110.

13. Napolitano, L.A., et al. 2008. Growth hormone enhances thymic function in HIV-1-infected adults. J. Clin. Invest. 118:1085-1098.

14. Napolitano, L.A., et al. 2002. Increased thymic mass and circulating naive CD4 T cells in HIV-1-infected adults treated with growth hormone. AIDS. 16:1103-1111.

15. Lo, J.C., et al. 2004. The effects of low-dose growth hormone in HIV-infected men with fat accumulation: a pilot study. Clin. Infect. Dis. 39:732-735.

16. Hazenberg, M.D., et al. 2004. Establishment of the CD4+ T-cell pool in healthy children and untreated children infected with HIV-1. Blood. 104:3513-3519.

17. Haynes, B.F., et al. 1999. Analysis of the adult thymus in reconstitution of $\mathrm{T}$ lymphocytes in HIV-1 infection. J. Clin. Invest. 103:453-460.

18. Connors, M., et al. 1997. HIV infection induces changes in $\mathrm{CD}^{+} \mathrm{T}$-cell phenotype and depletions within the $\mathrm{CD} 4^{+} \mathrm{T}$ cell repertoire that are not immediately restored by antiviral or immune-based therapies. Nat. Med. 3:533-540.

\title{
Discovering early molecular determinants of leukemogenesis
}

\author{
Grover C. Bagby \\ Departments of Medicine and Molecular and Medical Genetics, Oregon Health and Sciences University, \\ and Northwest Veterans Affairs Cancer Research Center, Portland, Oregon, USA.
}

\begin{abstract}
Truncating mutations of the G-CSF receptor are found during disease course in nearly half of all patients with severe congenital neutropenia. In this issue of the JCI, Liu et al. demonstrate that these mutations confer a competitive clonal advantage upon HSCs in mice and that the advantage is conditional because it is observed only in the presence of the ligand G-CSF (see the related article beginning on page 946). Once activated, the mutant receptor requires the function of Stat 5 in order to effect clonal expansion of this stem cell population. The results support the notion that early molecular steps in this and other neoplastic processes represent adaptations in which, through somatic mutations, "unfit" stem cells gain a measure of fitness by altering their relationships with their microenvironment.
\end{abstract}

Advances in biotechnology and genomics have catalyzed enormously important discoveries in the field of cancer biology. Some of the findings from studies of molecular pathogenesis have led to the development of new therapeutic agents that have notably controlled the growth of malignant

Nonstandard abbreviations used: AML, acute myeloid leukemia; ELA2, elastase 2; FA, Fanconi anemia; MDS, myelodysplastic syndrome; SCN, severe congenital neutropenia.

Conflict of interest: The author has declared that no conflict of interest exists.

Citation for this article: J. Clin. Invest. 118:847-850 (2008). doi:10.1172/JCI35109. cells in vivo (1). Truly targeted therapy is that which effectively interdicts a survival or replication pathway on which the malignant cell depends but normal cells do not. To develop this kind of therapeutic agent requires that one first identify such a defect in a malignant cell population, then develop an agent that attacks it in a specific way. The target must also be validated in clinical trials. That is, clinical responses must be attributable to the capacity of the therapeutic agent to interdict the function of the target molecule in the neoplastic cells.

Not only have advances in molecularly targeted therapy saved thousands of lives
(1), the successes to date have validated the simple principle that if you understand it you can fix it. For cancer therapeutics this idea is widely accepted today and is supported by massive investments in this approach by pharmaceutical and biotechnology firms. However, while few would argue with the idea that preventing cancer is better than treating it, investigators in the field of cancer prevention have not particularly warmed to the notion that the molecular strategies used for developmental therapeutics can support their goals as well. This situation has to change because there is clear-cut experimental evidence that the earliest control points for carcinogenic change in stem cells can be identified by focusing on stem cell pools under stress. The natural implication is that by interdicting this stress one might prevent the evolution of neoplastic clones.

\section{Stressed stem cell pools are vulnerable to neoplastic change}

Most leukemias evolve as clonal outgrowths of single, somatically mutated HSCs. Studies of patients with inherited 